\title{
Transcatheter Treatment of Hepatocellular Carcinoma with Doxorubicin-loaded DC Bead (DEBDOX): Technical Recommendations
}

\author{
Riccardo Lencioni - Thierry de Baere $\cdot$ Marta Burrel • James G. Caridi • \\ Johannes Lammer - Katerina Malagari • Robert C. G. Martin • Elizabeth O'Grady • \\ Maria Isabel Real · Thomas J. Vogl $\cdot$ Anthony Watkinson · Jean-Francois H. Geschwind
}

Received: 23 February 2011/ Accepted: 27 September 2011/Published online: 19 October 2011

(C) The Author(s) 2011. This article is published with open access at Springerlink.com

\begin{abstract}
Tranarterial chemoembolization (TACE) has been established by a meta-analysis of randomized controlled trials as the standard of care for nonsurgical patients with large or multinodular noninvasive hepatocellular carcinoma (HCC) isolated to the liver and with preserved liver function. Although conventional TACE with administration of an anticancer-in-oil emulsion followed by embolic agents has been the most popular technique, the introduction of embolic drug-eluting beads has provided an alternative to lipiodol-based regimens. Experimental studies have shown that TACE with drug-eluting beads has a safe pharmacokinetic profile and results in effective tumor killing in animal models. Early clinical experiences have
\end{abstract}

\section{R. Lencioni $(\square)$}

Division of Diagnostic Imaging and Intervention, Pisa University

Hospital, University of Pisa, Building No. 29, 2nd floor,

Via Paradisa 2, 56124 Pisa, IT, Italy

e-mail: riccardo.lencioni@med.unipi.it

T. de Baere

Department of Interventional Radiology, Institut Gustav-Roussy,

114 rue Édouard-Vaillant, 94805 Villejuif Cedex, France

\section{Burrel · M. I. Real}

Department of Radiology, Barcelona Clinic for Liver Cancer,

Hospital Clinic, Villarroel 170, 08036 Barcelona, Spain

J. G. Caridi

Division of Interventional Radiology, University of Florida,

P.O. Box 100374, Gainesville, FL 32610-0374, USA

\section{J. Lammer}

Department of Interventional Radiology, Medical University of Vienna, Guertel 18-20, 1090 Vienna, Austria

K. Malagari

Department of Radiology, University of Athens,

Papadiamandopoulou Street, Ilisia, 11528 Athens, Greece confirmed that drug-eluting beads provide a combined ischemic and cytotoxic effect locally with low systemic toxic exposure. Recently, the clinical value of a TACE protocol performed by using the embolic microsphere DC Bead loaded with doxorubicin (DEBDOX; drug-eluting bead doxorubicin) has been shown by randomized controlled trials. An important limitation of conventional TACE has been the inconsistency in the technique and the treatment schedules. This limitation has hampered the acceptance of TACE as a standard oncology treatment. Doxorubicin-loaded DC Bead provides levels of consistency and repeatability not available with conventional TACE and offers the opportunity to implement a

\section{R. C. G. Martin}

Division of Surgical Oncology, University of Louisville, 315 East Broadway, Suite 312, Louisville, KY 40202, USA

E. O’Grady

Department of Radiology, University Hospital Aintree,

Longmoor Lane, L9 7AL Liverpool, UK

T. J. Vogl

Department of Radiology, University of Frankfurt, Frankfurt Theodor-Stern-Kai 7, 60590 Frankfurt am Main, Germany

\author{
A. Watkinson \\ Department of Interventional Radiology, Royal Devon and \\ Exeter Hospital, Barrack Road, EX2 5DW Exeter, UK \\ J.-F. H. Geschwind \\ Division of Interventional Radiology, Johns Hopkins University, \\ 600 North Wolfe Street, Baltimore, MD 21287, USA
}


standardized approach to HCC treatment. With this in mind, a panel of physicians took part in a consensus meeting held during the European Conference on Interventional Oncology in Florence, Italy, to develop a set of technical recommendations for the use of DEBDOX in HCC treatment. The conclusions of the expert panel are summarized.

Keywords Chemoembolization - Doxorubicin · Drug-eluting bead $\cdot$ Hepatocellular carcinoma

\section{Introduction}

Hepatocellular carcinoma (HCC) is the sixth most common cancer and the third leading cause of cancer-related death [1]. Unlike most solid cancers, future incidence and mortality rates for HCC were projected to largely increase in several regions around the world over the next 20 years $[2,3]$. A careful multidisciplinary assessment of tumor characteristics, liver function, and physical status is required for proper therapeutic management of $\mathrm{HCC}[4,5]$.

Transarterial chemoembolization (TACE) has been established by a meta-analysis of randomized controlled trials as the standard of care for nonsurgical patients presenting with large or multinodular noninvasive tumor isolated to the liver and preserved liver function [6]. TACE is also used in patients with early-stage HCC when curative therapies-including liver transplantation, hepatic resection, and image-guided ablation-are precluded as well as in the setting of combination strategies including transcatheter and percutaneous treatments [7-12].

The ideal TACE scheme should allow maximum and sustained concentration of the chemotherapeutic drug within the tumor with minimal systemic exposure combined with calibrated tumor vessel obstruction [13]. Although conventional TACE with administration of an anticancer-in-oil emulsion followed by embolic agents has been the most popular technique, the introduction of embolic, drug-eluting beads has provided an attractive alternative to lipiodol-based regimens [11, 14, 15]. Experimental studies have shown that TACE with drugeluting beads has a safe pharmacokinetic profile and results in effective tumor killing in animal models [16-18]. Early clinical experiences have confirmed that drug-eluting beads provide a combined ischemic and cytotoxic effect locally with low systemic toxic exposure [19-24].

Recently, the clinical value of a TACE protocol performed by using the embolic microsphere DC Bead (Biocompatibles, UK) loaded with doxorubicin (DEBDOX; drug-eluting bead doxorubicin) has been shown by randomized controlled trials. In particular, in a multicenter study including 201 European patients (PRECISION V), use of DEBDOX resulted in a marked and statistically significant reduction in liver toxicity and drug-related adverse events compared with conventional TACE with lipiodol and doxorubicin $[25,26]$. Contrary to the observation in the conventional TACE arm, high-dose doxorubicin treatment could be applied according to the planned schedule in the whole DEBDOX group, resulting in consistently high rates of objective response and disease control in all preplanned subgroup analyses [25]. Two other trials reported higher rates of tumor response and longer time to progression for the loaded DC Bead as compared to a bland embolic microsphere with similar characteristics [27, 28]. As a result of these investigations, DEBDOX has been increasingly used as the first-line transcatheter treatment for HCC [2932].

An important limitation of conventional TACE has been the inconsistency in the technique and the treatment schedules. This limitation has greatly hampered the acceptance of TACE as a standard oncology treatment. DEBDOX provides levels of consistency and repeatability not available with conventional TACE, and offers the opportunity to implement a standardized approach to HCC treatment. With this in mind, a panel of physicians took part in a consensus meeting held during the European Conference on Interventional Oncology in Florence, Italy, to develop a set of technical recommendations for the use of DEBDOX in HCC treatment. The conclusions of the expert panel are summarized here.

\section{Technical Recommendations for the Use of DEBDOX in HCC Treatment}

The technical recommendations that are presented in this document represent the consensus of a panel of experts, all of whom have experience with the use of DEBDOX in HCC treatment. However, although these recommendations may be used as general guide to the use of DEBDOX in HCC treatment, the interventional radiologist treating the patient is the only physician who can decide how to approach the unique combination of patient and tumor characteristics that he or she is facing at the time of the procedure.

\section{Pretreatment Imaging}

Obtaining a triple-phase computed tomography (CT) or magnetic resonance imaging of the liver is required to integrate clinical and laboratory data to evaluate the indication to transcatheter treatment of HCC with DEBDOX in each individual patient by the local multidisciplinary liver tumor board. Additional imaging examinations to exclude extrahepatic disease should be performed as appropriate. 
Periprocedure Medication

Pain medication should be provided according to standard hospital protocols. Antibiotic prophylaxis and gastric protection should be administered at the physician's discretion.

\section{Loading Dose of Doxorubicin}

Each vial of DC Bead ( $2 \mathrm{ml}$ of beads) should be loaded with 50-75 mg doxorubicin (loading dose, 25-37.5 mg doxorubicin/ml of beads).

\section{Planned Dose of Doxorubicin}

The planned dose of doxorubicin should depend on the extent of the liver tumor burden. We acknowledge, however, that absolute recommendations cannot be issued in this regard, as individual patient- and tumor-related factors play an important role in the decision. As a general rule, different doses are recommended for patients with limited disease (defined as HCC within Milan criteria for liver transplantation: single tumor $\leq 5 \mathrm{~cm}$ or multiple tumors (up to 3 ) $\leq 3 \mathrm{~cm}$ each, or more advanced disease.

For disease within the Milan criteria, as a general rule, each single treatment should include a planned dose of up to $75 \mathrm{mg}$ doxorubicin loaded into one vial of DC Bead. For disease beyond the Milan criteria, as a general rule, each single treatment should include a planned dose of up to $150 \mathrm{mg}$ doxorubicin loaded into two vials of DC Bead.

In bilobar tumors, the two hepatic lobes can be treated in separate treatment sessions 2-4 weeks apart, in the absence of complications requiring a longer time interval between the two sessions. Obtaining confirmation that the liver enzymes have returned to baseline before performing the second treatment session is recommended. Treatment of both hepatic lobes in the same treatment session is possible in properly selected candidates if adequate interventional and clinical expertise is in place. In this case, the dose will be split according to the extent of the tumor burden in each lobe.

In very large tumors, even if unilobar, the same approach including two sessions should be followed, as a general rule. Indication to transcatheter treatment with DEBDOX in patients with tumor replacing more than $50 \%$ of the liver parenchyma, however, should be carefully evaluated. Adequate interventional and clinical expertise is required to manage patients with such advanced disease.

\section{Choice of DC Bead Size}

Use of 100-300 $\mu \mathrm{m}$ beads is recommended for a standard procedure. This choice is based on the demonstration that such small particles are delivered inside the tumor or in close proximity to the tumor margin and thus are ideal for drug delivery or precise embolization [33]. However, individual patient and tumor characteristics, particularly the identification of arteriovenous shunting, should be taken into account when the safety of the treatment and the choice of DC Bead size are determined. In the case of significant arterioportal or hepatic venous shunting, embolization of the shunt with gelfoam pledgets is recommended before proceeding with DEBDOX administration. Angiographic confirmation that the shunt is no longer present must be obtained before DEBDOX injection can be performed, and a larger bead size may be preferred.

\section{Bead Dilution}

Loaded DC Bead should be mixed with a nonionic contrast medium. At least 5-10 ml of nonionic contrast should be used per $1 \mathrm{ml}$ of DC Bead (i.e., 10-20 ml are required to dilute one vial of DC Bead) before injection. A good suspension of DC Bead in the contrast should be ensured before delivery.

\section{Catheter Positioning}

A superselective (i.e., segmental or subsegmental) approach should be used whenever possible by using a microcatheter. Use of 3D multiplanar reconstructions (MPR) obtained from C-arm rotational angiography with a flat-panel detector system (cone-beam CT) is recommended, if available, to improve the accuracy in identifying tumor-feeding arteries [34-36]. In addition, repeat cone beam CT is recommended after successful delivery of the DC Bead to confirm adequate targeting and saturation of the tumor(s).

For the segmental/subsegmental approach, the microcatheter is placed distally in the segmental/subsegmental tumor feeding vessel while ensuring that there is sufficient flow to the tumor. The clinician should avoid wedging the catheter to prevent reflux along the catheter shaft.

For the lobar approach, the catheter should be placed as selectively as possible in the right or left hepatic artery, with the clinician paying attention to identifying the origin of the cystic artery as well as other arteries supplying flow to extrahepatic organs. If identified, these vessels must be either embolized using coils or avoided by placing the catheter tip well beyond the origin of these vessels. In addition, forward flow into the desired vessel must be maintained because inadvertent administration or reflux of DC Bead into these extrahepatic vessels would be undesirable. 
Injection Rate

The injection must be very slow. An injection rate of $1 \mathrm{ml}$ of the contrast agent-DC Bead suspension per minute is recommended. Care should be taken to avoid sedimentation of the beads in the syringe by rotating the syringes or using a three-way stopcock to gently suspend the beads in the solution.

\section{Embolization End Point}

Injection should be continued until near stasis is observed in the artery directly feeding the tumor (i.e., the contrast column should clear within 2-5 heartbeats). At that point, injection must be stopped, regardless of the amount of beads that have been actually administered, to avoid reflux of embolic material. Once the embolization end point has been achieved, no additional embolic material should be injected.

If the near-stasis end point is not obtained after injection of the scheduled volume of loaded beads, two different options are possible. One option is to inject additional unloaded beads until the embolization end point has been reached. Another option is to not inject additional unloaded beads and to schedule the patient for a repeat course of treatment after imaging follow-up. This second option was supported by most panelists. However, there are insufficient data to mandate one strategy over the other.

\section{Posttreatment Imaging}

Obtaining a triple-phase CT or magnetic resonance imaging of the liver 2-4 weeks after treatment is recommended to assess tumor response and to plan further action. The panel recommends the use of the modified Response Evaluation Criteria in Solid Tumors (mRECIST) for HCC guideline for response classification [37]. Tumor response measured by mRECIST after transcatheter or systemic therapy has been shown to be associated with survival $[38,39]$.

In patients with residual viable tumor-including partial response, stable disease, and progressive disease according to mRECIST - further treatment with DEBDOX can be scheduled after 4-8 weeks in the absence of contraindications. Obtaining confirmation that the liver enzymes have returned to baseline before repeating treatment is recommended.

In patients with no evidence of residual viable diseasei.e., with complete response according to mRECISTimaging follow-up should be scheduled every 2-3 months.

\section{Treatment Discontinuation}

Treatment with DEBDOX should be discontinued-even if technically feasible-in patients presenting with untreatable progression [40]. Untreatable progression is defined as failure to achieve objective response in the targeted tumor after at least two DEBDOX treatments. The emergence of new intrahepatic tumor foci remote from the treated territory, although clearly representing tumor progression according to mRECIST for HCC, does not contraindicate further treatment with DEBDOX. In cases of clinical or functional deterioration, treatment should be discontinued in patients who have clinical progression to ECOG performance status $>2$ or who experience evolution to sustained hepatic decompensation (not merely after therapy).

\section{Final Remarks}

The technical recommendations that we summarize here represent the consensus of a panel of experts and are aimed at defining standards for an appropriate and consistent use of DEBDOX in the treatment of HCC. However, given the many patient- and tumor-related variables that play a role in the decision-making process, this is intended as no more than a general guideline. We fully acknowledge that, given the complexity of HCC, individual patient and tumor characteristics may require a different approach. Interventional radiologists should not follow these technical recommendations if, in their opinion, a different approach is required for the individual patient.

Finally, despite the improved tolerability profile of DEBDOX with respect to conventional TACE, it is imperative that interventional radiologists are fully aware of the spectrum of potential adverse events associated with the procedure to prevent complications or manage them properly [41].

Acknowledgment The authors thank the Research Analysis Library for assistance in the literature review and in the preparation and revision of the article, based on our detailed feedback. Editorial assistance was supported by Biocompatibles UK Limited.

Conflict of interest The authors declare that they have no conflict of interest.

Open Access This article is distributed under the terms of the Creative Commons Attribution Noncommercial License which permits any noncommercial use, distribution, and reproduction in any medium, provided the original author(s) and source are credited.

\section{References}

1. Parkin DM, Bray F, Ferlay J, Pisani P (2005) Global cancer statistics, 2002. CA Cancer J Clin 55:74-108

2. Olsen AH, Parkin DM, Sasieni P (2008) Cancer mortality in the United Kingdom: projections to the year 2025. Br J Cancer 99: 1549-1554 
3. Davis GL, Alter MJ, El-Serag H et al (2010) Aging of the hepatitis C virus (HCV)-infected persons in the United States: a multiple cohort model of HCV prevalence and disease progression. Gastroenterology 138:513-521

4. Bruix J, Sherman M (2005) Management of hepatocellular carcinoma. Hepatology 42:1208-1236

5. Llovet JM, Di Bisceglie AM, Bruix J et al (2008) Panel of experts in HCC-design clinical trials. Design and endpoints of clinical trials in hepatocellular carcinoma. J Natl Cancer Inst 100: 698-711

6. Llovet JM, Bruix J (2003) Systematic review of randomized trials for unresectable hepatocellular carcinoma: chemoembolization improves survival. Hepatology 37:429-442

7. Vogl TJ, Zangos S, Balzer JO et al (2007) Transarterial chemoembolization (TACE) in hepatocellular carcinoma: technique, indication and results. Forschr Geb Roentgenstr 179:1113-1126

8. Lencioni R, Crocetti L, Petruzzi P et al (2008) Doxorubicineluting bead-enhanced radiofrequency ablation of hepatocellular carcinoma: a pilot clinical study. J Hepatol 49:217-222

9. Vogl TJ, Nabuib NN, Nour-Eldin NE et al (2009) Review on transarterial chemoembolization in hepatocellular carcinoma: palliative, combined, neoadjuvant, bridging, and symptomatic indications. Eur J Radiol 72:505-516

10. Liapi E, Geschwind JF (2010) Intra-arterial therapies for hepatocellular carcinoma: Where do we stand? Ann Surg Oncol 17: 1234-1246

11. Lencioni R (2010) Loco-regional treatment of hepatocellular carcinoma. Hepatology 52:762-773

12. Raoul JL, Sangro B, Forner A et al (2011) Evolving strategies for the management of intermediate-stage hepatocellular carcinoma: available evidence and expert opinion on the use of transarterial chemoembolization. Cancer Treat Rev 37:212-220

13. Brown DB, Gould JE, Gervais DA et al (2009) Society of interventional radiology technology assessment committee, the international working group on image-guided tumor ablation. Transcatheter therapy for hepatic malignancy: standardization of terminology and reporting criteria. J Vasc Interv Radiol 20 (7 suppl):S425-S434

14. Kettenbach J, Stadler A, Katzler IV et al (2008) Drug-loaded microspheres for the treatment of liver cancer: review of current results. Cardiovasc Intervent Radiol 31:468-476

15. Liapi E, Geschwind JF (2011) Transcatheter arterial chemoembolization for liver cancer: Is it time to distinguish conventional from drug-eluting chemoembolization? Cardiovasc Intervent Radiol 34:37-49

16. Hong K, Khwaja A, Liapi E, Torbenson MS, Georgiades CS, Geschwind JF (2006) New intra-arterial drug delivery system for the treatment of liver cancer: preclinical assessment in a rabbit model of liver cancer. Clin Cancer Res 12:2563-2567

17. Lee KH, Liapi EA, Cornell C et al (2010) Doxorubicin-loaded QuadraSphere microspheres: plasma pharmacokinetics and intratumoral drug concentration in an animal model of liver cancer. Cardiovasc Intervent Radiol 33:576-582

18. Jordan O, Denys A, De Baere T, Boulens N, Doelker E (2010) Comparative study of chemoembolization loadable beads: in vitro drug release and physical properties of DC bead and hepasphere loaded with doxorubicin and irinotecan. J Vasc Interv Radiol 21:1084-1090

19. Varela M, Real MI, Burrel M et al (2007) Chemoembolization of hepatocellular carcinoma with drug eluting beads: efficacy and doxorubicin pharmacokinetics. J Hepatol 46:474-481

20. Poon RT, Tso WK, Pang RW et al (2007) A phase I/II trial of chemoembolization for hepatocellular carcinoma using a novel intra-arterial drug-eluting bead. Clin Gastroenterol Hepatol 5:1100-1108
21. Malagari K, Chatzimichael K, Alexopoulou E et al (2008) Transarterial chemoembolization of unresectable hepatocellular carcinoma with drug eluting beads: results of an open-label study of 62 patients. Cardiovasc Intervent Radiol 31:269-280

22. Grosso M, Vignali C, Quaretti P et al (2008) Transarterial chemoembolization for hepatocellular carcinoma with drugeluting microspheres: preliminary results from an Italian multicentre study. Cardiovasc Intervent Radiol 31:1141-1149

23. Reyes DK, Vossen JA, Kamel IR et al (2009) Single-center phase II trial of transarterial chemoembolization with drug-eluting beads for patients with unresectable hepatocellular carcinoma: initial experience in the United States. Cancer J 15:526-532

24. Seki A, Hori S, Kobayashi K, Narumiya S (2011) Transcatheter arterial chemoembolization with epirubicin-loaded superabsorbent polymer microspheres for 135 hepatocellular carcinoma patients: single-center experience. Cardiovasc Intervent Radiol 34:557-565

25. Lammer J, Malagari K, Vogl T et al (2010) Prospective randomised study of doxorubicin-eluting-bead embolization in the treatment of hepatocellular carcinoma: results of the PRECISION V study. Cardiovasc Intervent Radiol 33:41-52

26. Vogl TJ, Lammer J, Lencioni R et al (2011) Liver, gastrointestinal, and cardiac toxicity in intermediate hepatocellular carcinoma treated with Precision TACE with drug-eluting beads: results from the PRECISION V randomized trial. AJR Am J Roentgenol 197:W562-W570

27. Malagari K, Pomoni M, Kelekis A et al (2010) Prospective randomized comparison of chemoembolization with doxorubicineluting beads and bland embolization with Beadblock for hepatocellular carcinoma. Cardiovasc Intervent Radiol 33:541-551

28. Nicolini A, Martinetti L, Crespi S, Maggioni M, Sangiovanni A (2010) Transarterial chemoembolization with epirubicin-eluting beads versus transarterial embolization before liver transplantation for hepatocellular carcinoma. J Vasc Interv Radiol 21: 327-332

29. Sadick M, Haas S, Loehr M et al (2010) Application of DC beads in hepatocellular carcinoma: clinical and radiological results of a drug delivery device for transcatheter superselective arterial embolization. Onkologie 33:31-37

30. Martin R, Irurzun J, Munchart J et al (2011) Optimal technique and response of doxorubicin beads in hepatocellular cancer: bead size and dose. Korean J Hepatol 17:51-60

31. Namur J, Citron SJ, Sellers MT et al (2011) Embolization of hepatocellular carcinoma with drug-eluting beads: doxorubicin tissue concentration and distribution in patient liver explants. $\mathrm{J}$ Hepatol [Epub ahead of print]

32. Song MJ, Park CH, Kim JD et al (2011) Drug-eluting bead loaded with doxorubicin versus conventional lipiodol-based transarterial chemoembolization in the treatment of hepatocellular carcinoma: a case-control study of Asian patients. Eur J Gastroenterol Hepatol 23:521-527

33. Lee KH, Liapi E, Vossen JA et al (2008) Distribution of iron oxide-containing embosphere particles after transcatheter arterial embolization in an animal model of liver cancer: evaluation with MR imaging and implication for therapy. J Vasc Interv Radiol 19:1490-1496

34. Kim HC, Chung JW, Park JH et al (2009) Transcatheter arterial chemoembolization for hepatocellular carcinoma: prospective assessment of the right inferior phrenic artery with C-arm CT. J Vasc Interv Radiol 20:888-895

35. Miyayama S, Yamashiro M, Okuda M et al (2009) Usefulness of cone-beam computed tomography during ultraselective transcatheter arterial chemoembolization for small hepatocellular carcinomas that cannot be demonstrated on angiography. Cardiovasc Intervent Radiol 32:255-264 
36. Miyayama S, Yamashiro M, Hattori Y et al (2011) Efficacy of cone-beam computed tomography during transcatheter arterial chemoembolization for hepatocellular carcinoma. Jpn J Radiol 29:371-377

37. Lencioni R, Llovet JM (2010) Modified RECIST (mRECIST) assessment for hepatocellular carcinoma. Semin Liver Dis 30: $52-60$

38. Edeline J, Boucher E, Rolland Y et al (2011) Comparison of tumor response by response evaluation criteria in solid tumors (RECIST) and modified RECIST in patients treated with sorafenib for hepatocellular carcinoma. Cancer [Epub ahead of print]
39. Gillmore R, Stuart S, Kirkwood A et al (2011) EASL and mRECIST responses are independent prognostic factors for survival in hepatocellular cancer patients treated with transarterial embolisation. J Hepatol [Epub ahead of print]

40. Lencioni R, Zou J, Leberre M et al (2010) Sorafenib (SOR) or placebo (PL) in combination with transarterial chemoembolization (TACE) for intermediate-stage hepatocellular carcinoma (SPACE) (abstract TPS178). J Clin Oncol 28(suppl):15s

41. Malagari K, Pomoni M, Spyridopoulos TN et al (2011) Safety profile of sequential transcatheter chemoembolization with DC Bead: results of 237 hepatocellular carcinoma (HCC) patients. Cardiovasc Intervent Radiol 34:774-785 